\title{
The Recovery Room: Transition from a Sleepy Postoperative Unit to a Vibrant and Cost-Effective Multipurpose Perioperative Care Unit
}

\author{
Stefan Schad' \\ Hendrik Booke ${ }^{2}$ \\ Serge C Thal (iD ${ }^{3}$ \\ Alexander $\mathrm{H}$ Bentley $^{3}$ \\ Michael Booke $\mathbb{D}^{\prime}$ \\ 'Department of Anesthesiology, Intensive \\ Care Medicine and Pain Therapy, \\ Kliniken-MTK, Academic Hospital of the \\ Goethe-University Frankfurt, Frankfurt, \\ Germany; ${ }^{2}$ Department of \\ Anesthesiology, Intensive Care Medicine \\ and Pain Therapy, University Hospital \\ Frankfurt, Goethe University, Frankfurt, \\ Germany; ${ }^{3}$ Department of \\ Anesthesiology, HELIOS University \\ Hospital Wuppertal, Witten/Herdecke \\ University, Wuppertal, Germany
}

\begin{abstract}
The anesthesiologist, who traditionally was solely responsible for the intra- and postoperative care of patients, has undergone a transformation over the last decades and has emerged as a specialist for perioperative medicine. This includes preoperative assessment, preoperative stabilization of emergent cases, pre- or postoperative initiation of regional blocks, postoperative recovery and if needed postoperative intensive care outside the intensive care unit. A traditional recovery room, designated to take care of patients emerging from anesthesia only, no longer matches the modern anesthesiologist's demands. However, a traditional recovery room can easily be transformed into a vibrant multi-purpose perioperative care unit. Especially in smaller hospitals, this serves to match the anesthesiologist's demands without the financial burden of separate units for each task. On the contrary, it allows to transform the recovery room from a mandatory, but costly postoperative unit into a highly productive and demanding perioperative unit, allowing for extra revenues without corresponding costs. Worldwide, operating rooms are linked to an adjacent recovery room allowing patients to emerge from anesthesia until they fulfill the criteria to be transferred either to the regular ward or, in case of outpatient surgery, to be discharged home. Running these recovery rooms, however, is expensive due to the required technical equipment and the monthly costs of highly qualified anesthesia personnel. Despite these financial burdens, such recovery rooms are still mandatory to ensure full recovery after anesthesia and surgery. In most countries, there is no (full) reimbursement for providing recovery rooms, turning them into fiscally deficient units in most hospitals. However, recovery rooms can be further developed allowing hospitals to improve their caseloads, reduce turnover times in the operating room, and even help to manage a shortage of beds in the intensive care unit. In this paper, we describe the potential transformation from a traditional recovery room to a multi-purpose perioperative high-tech unit.
\end{abstract}

Keywords: recovery room, postanesthesia care unit, perioperative medicine, turnover time, reimbursement, same-day admission, holding area

\section{Expert Opinion}

With the development of professional anesthesiology in the mid-twentieth century, not only the number of operations but also the complexity and duration of surgical procedures increased dramatically. This in turn required deeper and longer general anesthesia. A recovery room was soon mandatory, as patients required special postoperative care to manage pain while simultaneously slowly emerging from anesthesia. ${ }^{1}$

Progress in pharmaceutical research has brought about anesthetics, narcotics, and neuromuscular blocking agents with shorter half-lives, especially in the last 
two decades, allowing for improved turnover times in the operating room (OR). ${ }^{2}$ Short-acting and nonaccumulating narcotics like remifentanil, anesthetics like propofol or desflurane, and non-depolarizing muscle relaxants like cisatracurium served to reliably terminate anesthesia after surgery. Patients are nowadays transferred to the recovery room in an awake state rather than in deep sedation, and residual anesthesia is rarely seen. Simultaneously, the time needed to monitor postoperative patients has decreased, and most patients can be safely transferred to the regular ward within 30 to 45 minutes postoperatively. This opens the door to additional recovery room duties. The anesthesiologist, who has evolved into a specialist for perioperative medicine in the last decades, ${ }^{3}$ has the expertise and motivation to cover these extra duties.

\section{Preoperative Holding Area}

Operating suites built nowadays are designed with an adjacent holding area. This area serves to have the patient waiting in the immediate vicinity of the OR, thereby avoiding any delays due to patient transport from the regular ward. ${ }^{4}$ With the OR being the most expensive unit of hospitals, idle time is to be avoided by any means.

Operating suites older than 10 to 15 years have no extra holding area, and most of them simply do not have space to implement one. In these cases, the recovery room may serve as such. The only disadvantage is that patients waiting for their operation come in contact with postoperative patients. However, this can easily be mitigated by spatial separation and experienced recovery room nurses.

We also use the recovery room for the first cases of the day, guaranteeing a punctual start in each OR. Therefore, all patients scheduled first are transferred to the recovery room 30 to 45 minutes prior to surgery. At this time of the day, no postoperative patients are present in the recovery room, and hence, there is enough capacity to accommodate all first-case patients. This also leaves enough time to review all preoperative checklists. Some authors define this as a preoperative assessment clinic (PAC) and have this entity established as a separate unit. ${ }^{5}$ We believe this is too resource-intensive and have integrated the PAC as part of our recovery room with no extra staff costs.

\section{Preoperative Same Day Admission Center}

Same-day admissions are beneficial because they spare the preoperative day in hospital, thus reducing hospital costs. Patients are to be admitted to the regular ward early in the morning, just prior to surgery. At that time, however, the wards are often still occupied by patients scheduled for discharge on that day, putting undue pressure on the nurses and leaving the patients confused and dissatisfied. To improve work-flow, some hospitals have opened special admission centers for same-day surgery without compromising surgical outcome. ${ }^{6,7}$ We thus decided to further optimize the admission process: part of our recovery room is now used for same-day admissions. After arrival, patients can change into a hospital gown prior to surgery and are then assigned a bed within the recovery room, where pre- and postoperative patients are separated using partition walls. From here, the process is equivalent to that of the holding area. The patient's belongings are put into a sealed box, which is transferred to the assigned ward while the patient undergoes surgery. Postoperatively, the patient is brought to our recovery room again and is then discharged to the regular ward, where a bed is now available.

\section{Preoperative Emergency Unit}

Facing restrictive capacities of the Emergency Department, the scope of tasks for the traditional recovery room can be expanded to improve the patient's condition preoperatively. ${ }^{8}$ Patients being admitted through the emergency department are transferred to our recovery room as soon as the decision to perform emergency or urgent surgery has been made. The patient can be stabilized and prepped with additional i.v. lines, central venous catheters and/or arterial lines while waiting for surgery. On completion, the patient is ready to be transferred to the next available OR without delay, and surgery can commence immediately after induction of anesthesia.

\section{Preoperative Regional Anesthesia Center}

Patients scheduled for surgery but in need of regional anesthesia prior to induction of anesthesia are transferred to the recovery room, where the attending anesthesiologist performs the necessary procedure. This allows for improved turnover time. Again, those patients are separated from postoperative patients using partition walls.

\section{Postoperative Anesthetic Care Unit (PACU)}

Patients undergoing major surgery may require elective postoperative intensive care. However, these plans are often subject to change due to unscheduled emergencies. ${ }^{8}$ 
In such cases, the recovery room can easily serve as a buffer as it is equipped like an intensive care unit (ICU), in addition to the attending anesthesiologist and anesthetic nurse being qualified to initiate postoperative and surgical intensive care. As soon as an ICU bed is available, the patient is transferred. Occasionally, during their PACU stay, some patients recover to the extent that allows for direct transfer to the regular ward a few hours later.

\section{Limitations}

As mentioned initially, the time needed for patients to emerge from anesthesia and to recover from surgical trauma has been shortened dramatically in recent years, leaving the recovery room with space and time-slots available for additional tasks. This also holds true for academic hospitals with their typical case mix, where some patients even nowadays need to spend some hours in the recovery room, while the majority is ready for discharge in less than an hour.

However, adding all of the duties mentioned above may overtax recovery room availability and may also overwhelm its personnel. This may even cause the recovery room to become a bottleneck for patients leaving the OR, resulting in increased turnover times. However, any such delay is counterproductive since it reduces the caseload per OR. ${ }^{9,10}$ Therefore, adequate planning of the OR schedule should incorporate the required level of postoperative care, taking into account the nursing staff level in the recovery unit and thus smoothing the workload for the staff personnel while preventing overtime hours. ${ }^{11}$ Further, it is imperative to manage the recovery room as strictly as the rest of the operating suite. Since the enhanced spectrum of the recovery room requires an anesthesiologist, it is preferable to have an experienced anesthesiologist rather than a surgeon or a nurse managing the operating suite and the recovery room. Further, anesthesiologists have a translational and multidisciplinary view necessary to manage such a modern and extended recovery room. Most, but not all, of our recovery room nurses have an anesthetic background. The overall number of nurses in the OR including the adjacent recovery room was not increased, but with the broadened spectrum of the recovery room, some nurses voluntarily relocated their daily job. Further, the number of patients fluctuates during the day, and so does the number of nurses in the recovery room, depending on the actual demand. Consecutively, the number of anesthetic nurses in the OR varies during the day, sometimes leaving one anesthetic nurse to cover two or rarely three ORs at a time. To allow for this needsbased allocation of nursing staff, each nurse was equipped with a mobile phone.

Quality of care, however, depends not only on the ratio of staff to patients but also on the hospitals' ability to promptly treat emergency patients. With our recovery room concept, we have added extra resources to meet this requirement.

Another limitation is hospital hygiene regulations, which apply to operating suites and cannot be ignored. Our recovery room concept has been approved by our hygiene commission and is in line with governmental hygiene legislation. According to German legislation, patients who are colonized or infected with multiresistant bacteria are to be separated from others. This also holds true for the recovery room, making it impossible to admit such patients during high-flow periods of the day. Our experience is that these rush hours for the recovery room cannot be predicted reliably. We thus schedule these patients at the end of each OR's surgical list, guaranteeing sufficient recovery room time for each patient in the OR itself.

\section{Conclusion}

Adding extra tasks to a standard recovery room easily goes along with the enhanced spectrum of anesthesiology. The holding area concept helps to improve turnover times and efficiency in each OR, allowing a higher throughput and thus more cases per day. The preoperative care of emergency patients as well as the PACU helps to increase the hospital's caseload and revenue, especially in case of emergency room and/or ICU overflow.

\section{Disclosure}

The authors report no conflicts of interest in this work.

\section{References}

1. Charbon HG, Livingstone HM. Planning a recovery room for adequate postoperative care. Hospitals. 1949;23:35-38.

2. White PF, Watcha MF. Pharmacoeconomics in anesthesia: what are the issues? Eur J Anesthesiol Suppl. 2001;23:10-15. doi:10.1046/j.13652346.2001.00010.x

3. Van Aken H, Thomson D, Smith G, et al. 150 years of anaesthesia a long way to perioperative medicine: the modern role of the anaesthesiologist. Eur J Anaesthesiol. 1998;15(5):520-523. doi:10.1097/ 00003643-199809000-00002

4. Sullivan EE. Preoperative holding areas. J Perianesth Nurs. 2000;15:353-354. doi:10.1053/jpan.2000.18207

5. Emanuel A, Macpherseon R. The anesthetic pre-admission clinic is effective in minimising surgical cancellation rates. Anaesth Intensive Care. 2013;41:90-94. doi:10.1177/0310057X1304100115 
6. Ziser A, Alkobi M, Markovits R, Rozenberg B. The postanaesthesia care unit as a temporary admission location due to intensive care and ward overflow. BJA. 2002;88:577-579. doi:10.1093/bja/88.4.577

7. Keränen J, Keränen U. From home to operation (FHTO - a new surgical admission centre: does the comprehensive initialisation of a new process harm outcome? Scand J Surg. 2011;110:136-140. doi:10.1177/145749691110000213

8. Prien T, van Aken $H$. The perioperative phase as a part of anesthesia. Anaesthesist. 1997;46:S109-S1013. doi:10.1007/PL00002470
9. Levine WC, Dunn PF. Optimizing operating room scheduling. Anesthesiol Clin. 2015;33(4):997. doi:10.1016/j.anclin.2015.07.006

10. van Veen-berk E, Elkhuizen SG, van Logten S, et al. Enhancement opportunities in operating room utilization; with a statistical appendix. J Surg Res. 2015;194(1):43-51. doi:10.1016/j.jss.2014.10.044

11. Debats CEJM, Dellaert NP, Pouwels S, et al. Balancing workload by using an integrated OR planning methodology. J Perianaesth Nurs. 2021;36:279-290. doi:10.1016/j.jopan.2020.09.004

\section{Publish your work in this journal}

ClinicoEconomics and Outcomes Research is an international, peerreviewed open-access journal focusing on Health Technology Assessment, Pharmacoeconomics and Outcomes Research in the areas of diagnosis, medical devices, and clinical, surgical and pharmacological intervention. The economic impact of health policy and health systems organization also constitute important areas of coverage. The manuscript management system is completely online and includes a very quick and fair peer-review system, which is all easy to use. Visit http://www.dovepress.com/testimonials.php to read real quotes from published authors. 